\title{
Comprehensive rehabilitation of herniated disc in the lumbar section of the spine.
}

\author{
Petya Kasnakova1, Anna Mihaylova ${ }^{1}$, Penka Petleshkova ${ }^{2 *}$ \\ ${ }^{1}$ Medical University of Plovdiv, Medical College, Plovdiv, Bulgaria \\ ${ }^{2}$ Department of Obstetrics and Gynecology, Plovdiv Medical University, Plovdiv, Bulgaria
}

\begin{abstract}
Herniated disc is one of the most common problems of the musculoskeletal system, and the spine, in particular. This article presents a modern algorithm of methods of treatment constituting comprehensive rehabilitation of herniated disc in the lumbar section of the spine, including: physical therapy, active and passive kinesitherapy, medical massage and manual techniques, applied in the medical practice.

Rehabilitation and physioprophylaxis facilitate the reduction of the risk of occurrence and development of herniated disc in the lumbar section of the spine through natural (water, air, movement and sunshine) and preformed physical factors, the main aim being achieving a good quality of life of both the sick and the healthy. The so called 'spine school', which includes training the patient to have a good posture, a good stance, to walk correctly and to improve his/her motor stereotype through control and self-control, plays a major role. In developing the individual treatment scheme for each patient at a certain stage of his/her condition, it is vital to attempt to achieve a synergic effect of the optimal combination of electrotherapeutic, peloid therapeutic and kinesitherapeutic methods.

Regular courses in physioprophylaxis and kinesio-prophylaxis significantly boost the effect of the medicamentous therapy in patients with herniated lumbar disc and improve their quality of life.
\end{abstract}

Keywords: Comprehensive rehabilitation, Herniated disc.

Accepted on July 17, 2018

\section{Introduction}

The spine is the main component of the axial system. In man, the spine is under exceptional strain because of the upright posture. It performs three main functions-supporting, dynamic and protective. From a mechanical point of view, these functions require opposing characteristics, which makes the spine an exceptionally overtaxed and delicate structure [1]. Its normal functioning depends on numerous interrelated factors, and any disturbance of the flexibility-stability balance causes adverse functional changes. The spine bears the weight of the head, the torso and the upper extremities, passing it via the pelvis to the lower extremities. It performs shock-absorbing movements ensuing from its specific shape and structure $[2,3]$. According to global statistics $80 \%-90 \%$ of the people have had backache at some time in their lives [4]. Such pain may be due to the great strain on the lumbar vertebrae and discs caused by the weight of the body, overweight, the adoption of a certain forced posture while working, watching TV, doing exercise or sport. The sedentary way of life, inadequate knowledge and prophylaxis of its treatment also have a significant impact [5].

Herniated lumbar disc is a medical, social and economic problem [6]. This condition affects people of all ages, and in many cases even young adolescents are diagnosed with it.
Epidemiological surveys show that the incidence is about $30 \%$, increasing after the age of $30 \mathrm{y}$ to reach its peak in patients aged 55-64 y [7]. It should be taken into account that CT of patients without any complaints established herniated disc, spinal stenosis and other degenerative changes in $50 \%$ of patients aged over $40 \mathrm{y}$. According to official data by 2014 , approximately $5 \%$ of the male and $2.5 \%$ of the female population had been diagnosed with herniated disc in advanced stage, to date, their percentage has probably increased. According to data provided by German author, Malchanova et al., one in twelve patients in the general practice, and one in three in the orthopaedic practices suffer from a lumbar syndrome. [8]

According to data of Kaemer, of 100 lumbar-radicular syndromes, 98 are treated conservatively, and only $2 \%$ undergo surgical treatment [9]. According to Kane, in the UK there are 15 surgical operations of herniated disc for 100 thousand people, and in some regions in the west parts of the USA, there are up to 80 surgical interventions for every 100 thousand people [8].

In fact, the herniated disc is a condition of the spine affecting the intervertebral disc. This is a condition in which the outer annulus fibrosus of the intervertebral disc is torn, thus allowing 
the soft central part-the pulpous nucleus-to prolapse in the spinal canal. The compression, most often affecting certain spinal cord nerves, causes the process of herniation of the pulpous nucleus, occurs as secondary pathology [10].

The risk factors include: anatomical features, microtraumatism, constitutional weakness and the age factor which, compounded with the listed above, is a leading factor in the pathology of the intervertebral disc [11].

The functions performed by the spine make it bear abrupt and big physical strain (carrying, lifting, etc.). These functions, its anatomical structure, as well as the vertical posture, are conducive to early degeneration of the intervertebral discs and discogenic diseases. The spine, even at rest, is subjected to various forces which cause slippage along its bone part or on the muscle-ligament part [1].

The herniated lumbar disc is the most common, usually affecting L4-L5 and L5-S1. The reason is to be found in the weight this part of the spine bears, incorrect motor skills and bad posture.

In the clinical symptoms of the herniated disc, there are three clearly differentiated and equal in importance pathological syndromes: vertebral syndrome, comprising pathological changes and the adoption of an antalgic posture as a result of the pain syndrome; neurological syndrome, characterized by affecting one or more roots, most often these are L5 and S1 and pain syndrome-local pain which can be constant, burning or root with distal radiation to the limb along the respective dermatome $[4,12]$.

The diagnosis of herniated disc is confirmed through a series of imaging tests: spondylography, CT, MRI [7,13].

\section{Treatment}

The treatment of herniated lumbar disc is a comprehensive one. The conservative therapy in most of the mild cases of disc protrusion, herniation in the early stage or a small herniation of the disc, usually gives good results. The treatment includes the intake of analgesics and antiedemic medicines; a period of rest; a special regimen of physical activity; physiotherapy and medical massage. Surgical intervention is necessary when the pain syndrome persists after the administered conservative therapy or the occurrence of neurological deficit expressed in significant motor weakness and pelvic reservoir dysfunction [14].

Adequate pain relief is the first and most important step in the treatment of back pain in cases of herniated disc. A combination of muscle relaxants and anti-inflammatory medicines; muscle relaxants are used to affect the spasms of paravertebral muscles, which greatly reduces the pain. They are administered when there is a spasm of the paravertebral muscle which can be palpated. The use of muscle relaxants boosts the clinical efficiency of the simultaneous intake of nonsteroid anti-inflammatory drugs. Non-steroid antiinflammatory drugs have an analgesic effect in small doses, and an anti-inflammatory effect in higher doses. The different drugs have different analgesic and anti-inflammatory action, and they start taking effect at different times. When the pain syndrome has become chronic, which is observed in $10 \%-15 \%$ of the patients, anti-depressants may be prescribed. Vitamins of group B are also included [15].

Practical experience shows that optimal treatment is impossible to achieve without comprehensive physical rehabilitation procedures. The chronically recurrent nature of the disease shows that it is not sufficient to treat only the severe backache. Instructions as to how to prevent the recurrence of the pain; in the sub-acute and chronic stage of the condition, physical therapy and rehabilitation are of vital importance. Kinesitherapy, with a wide range of means and methods, plays an important role in the prophylaxis of recurrent pain [16].

\section{Methods and Algorithms}

Methods and algorithms of physical prevention, therapy and rehabilitation of herniated disc; their aim is the recovery and stabilization of the functions of the lumbar-sacral section of the spine. The main purposes of the comprehensive rehabilitation are: improving and enhancing the psycho-emotional status of the patient; reducing the spasm and reflex rigidity of the paravertebral muscles; releasing the compressed nerve root and reducing lumbalgia (low back pain); improving muscular balance; preserving muscle tone and preventing muscle atrophy; eliminating any residual functional blockage; developing a healthy muscle corset; improving coordination and balance; developing correct posture and sitting stance while working; and prophylaxis of recurrences.

\section{Complex rehabilitation therapy}

The elaboration of an algorithm of a physical therapy rehabilitation complex is based on the main symptoms (pain symptoms, dynamic disturbances in the lumbar section of the spine), the stage and duration of the disease.

A detailed kinesiological analysis of the patient is made: physical and functional status with manual muscle testing and diagnostic tests and trials aimed at determining the rehabilitation potential [10].

During the acute stage of the condition, the main objective of the therapy is to reduce and eliminate the pain syndrome [17]. The means and methods used by medical massage are special reflex techniques, acupressure and zone therapy. The following physiotherapy techniques are included: electrophoresis with Novocain, interferential currents-initially with a frequency of 0-100 Hz (dynamogenic, trophic, spasmolytic and analgesic effect), dynamic currents-the modulations DF-2', CP and LP $2-5$ ' is used, magnetic therapy, ultra-sound with an intensity of 0.2-0.5 W/cm ${ }^{2}$. Kinesitherapy uses lighter techniques: positional therapy, mechanical therapy-the McKenzie method, respiratory and relaxing exercises, generally strengthening exercises only for the extremities without engaging the spine, isometric contractions, applied everyday exercises-the patient is taught how to get into and out of bed from lying sideways. 
In the sub-acute stage, the main objective is to mobilize the lumbar-sacral section of the spine. The aims include relieving the pain, maintaining the muscle trophics, relaxing and extending the contracted muscles, developing a sound muscle corset by boosting the abdominal and paravertebral muscles, training patients how to carry out correctly everyday activities.

In that stage, medical massage aims at releasing the nerve root, reducing the reactive inflammatory process around it and relaxing the paravertebral muscles. Underwater massage is also included [18].

In cases of established functional blockage of the intervertebral joints, degenerative changes of the spine, and lateral herniated disc, manual therapy is recommended. Manual therapy is an efficient method of treatment. Soft-tissue techniques, postisometric relaxation, mobilization, can be applied to release the compressed nerve root from the herniated disc, especially when the said methods are combined with extension therapy and manual traction techniques. Manual therapy, however, is absolutely counter-indicative in cases of median herniated discs [19].

From the range of physical therapy methods, electrophoresis with nivalin is administrated in cases of peripheral nerve damage to facilitate the passage in the synapse. The anode is placed in the area of the paretic muscles, and the cathode-in the area of the affected segment of the spine.

In the case of weakening motor function, electro-stimulation is applied on the damaged nerves and the respective innervated muscles. In the case of slightly damaged muscles, the ratio impulse/pause is $1: 1$, with duration of $10-20$ '. In cases of partial degeneration response the procedure begins with a frequency of $10-30 \mathrm{~Hz}$, impulse/pause 1:3. In cases of complete response of degeneration, the impulse is exponential in form, with a frequency of $0.5-10 \mathrm{~Hz}$, because the muscle tires easily and in 2-3' it needs a rest. The impulse/pause ratio has to be 1:6 [4].

In cases of expressed pain syndrome, a good pain-relief effect is achieved through TENS (transcutaneous electrical nerve stimulation)-impulse $300 \mu \mathrm{s}$, frequency $50-100 \mathrm{~Hz}$, with a duration of 15-20'. We administer interferential currents with a frequency of 90-100 Hz; low-frequency impulse magnetic field; sinusoidal modulated currents-in the event of pain syndrome, group III and IV are applied, $80 \mathrm{~Hz}$ and $50 \%$ depth of the modulation. When the pain symptoms recede, group III and IV are applied, $50-30 \mathrm{~Hz}, 75 \%-100 \%$ depth of the modulation, with a duration of $10-15^{\prime}$; ultraviolet radiationthree fields with the aim of relieving the pain (lumbar area, back surface of the thigh, and the back lateral area of the shin); laser therapy and laser puncture; thermal therapy [19].

From the range of kinesitherapeutic methods, extension therapy is applied at this stage, and it is imperative that the traction strength should be increased gradually; relaxation exercise in combination with respiratory exercise; traction exercise; de-blocking exercise; exercise for the abdominal muscles of Machek; Bankov's exercise; Lewit's and Zhelev's exercise; analytical exercise for the abductor and gluteus muscles; exercises designed to enhance the flexibility of the spine; exercises aimed at improving the gait and the posture; exercises aimed at improving balance; exercises for automobilization and against resistance. From lying on the back, the patient bends his/her lower limbs, embraces them with his/her arms and tries to straighten them against the resistance of the arms for 3-5 s [12].

In the chronic stage, the purpose of the comprehensive physical therapy and rehabilitation is to maintain the functional ability and efficiency of the patient in the long term. The therapy is designed to maintain the good overall physical conditions, to develop a functional muscle corset, to improve coordination and balance, to facilitate the normal performance of everyday tasks and habits, and to teach the patient to protect him/herself from recurrences.

The patient may be subjected to comprehensive rehabilitation therapy including: classical and medical massage, segment massage, periosteal massage and zone therapy for the purpose of relieving the pain in the lumbar-sacral area and the zones to which the pain radiates, improving blood circulation and trophics in this area, affecting in a reflective way the nerve structures, and in general strengthening the organism.

In this stage, physical therapy is aimed to prevent recurrences, to improve the trophics in the area of the damaged segment of the spine, to curb the expansion of connective tissue in the same area. Balneological factors are also made use ofhydrogen sulphide baths, radon baths and carbonic acidic baths. Suitable resorts in Bulgaria are: Pavel Banya, Kyustendil, Sapareva Banya, Pomorie, Momin Prohod, Marikostinovo, Velingrad. Mud baths are also beneficial.

As regards kinesitherapeutic methods, exercises aimed at improving the general development, in combination with respiratory exercises, coordination and balance exercises, analytical exercises in combination with isometric contractions and exercises of dynamic nature, exercises aimed at improving posture and gait, underwater exercises, everyday chores, special exercises for the paravertebral muscles, are highly recommended [12].

As means of prophylaxis of herniated disc, analytical exercises are beneficial, as well as sleeping on a hard bed, sitting upright, weight-lifting with straightened back, hiking, strengthening procedures, sport, sport elements, maintaining an optimal weight, leading a healthy way of life.

According to Zhelev et al., kinesitherapeutic methods appropriate for this stage include exercises and procedures aimed at improving lumbar stability, agility, flexibility, the functioning of the flexor and extensor muscles; exercises with a Swiss ball aimed at improving the proprioception of the spinal structures [20].

Each sport burdens and strains in a specific way and to a different degree the respective sections of the spine (Graciet et al.). In some cases patients have to give up certain sports, especially those that involve a high risk for the spine (e.g. 
rugby, weight-lifting, wrestling, wind-surfing, motocross, artistic gymnastics).

Principles of therapeutic approach: The therapeutic scheme should be independent and tailored to the respective patient. There should be no patterns in the therapeutic scheme, and certain methods should not be followed blindly. Herniated disc therapy has to start with physical therapy in combination with medicamentous therapy. The medicamentous therapy plays its role in the whole complex of comprehensive therapy, but it should not be given priority or favoured in any way. Physical medicine also has a preventive function, and in the case of this pathology, 3 to 4 courses of physical therapy a year are recommended [21].

\section{Conclusion}

It is recommended that all patients with herniated disc and backache should be administered comprehensive schedules of physical therapy and rehabilitation, provided by a multidisciplinary team of specialists (physician-neurologist, physiotherapist, rehabilitation therapist, ergotherapist, manual therapist, physiotherapeutic nurse, pharmacist and clinical psychologist). In developing the individual treatment scheme for each patient at a certain stage of his/her condition, it is vital to attempt to achieve a synergic effect of the optimal combination of electrotherapeutic, peloid therapeutic and kinesitherapeutic methods.

Regular courses in physioprophylaxis and kinesio-prophylaxis significantly boost the effect of the medicamentous therapy in patients with herniated lumbar disc and improve their quality of life.

In the event that conservative and physical therapy proves to be inefficient, and in the presence of absolute indications, then surgical treatment is recommended and administered. In the post-operative period, complex physiotherapy and rehabilitation procedures are mandatory.

\section{References}

1. Popov N. Functional features and prerequisites of pathokinetic changes in the cervical spine. Kinesitherapy Rehabilitation 2009; 3: 5.

2. Baltadzhiev G, Vassilev V, Baltadzhiev A. Anatomy of man. Plovdiv 2015.

3. Krastev D, Kalniov M, Krastev N. Anatomy of the musculoskeletal system with bases of biomechanics. Sofia 2012.

4. Koleva I. Algorithms for physical prevention, therapy and rehabilitation of some common and socially significant diseases. Sofia 2007.

5. Hoy D, Bain C, Williams G. A systematic review of the global prevalence of low back pain. Arthritis Rheum 2012; 2028-2037.
6. Zhelev V. Physiotherapy in neurological, neurosurgical and mental diseases. Sofia 2012.

7. Milanov I. Back pain (II Ed). Med Physical Education, Sofia 2010; 179.

8. Malchanova V. Vertebrae manipulations in the discordant PNC syndromes in the lumbar region of the spine. Candidate Dissertation, Sofia 1987.

9. Petrov P. Pain-disc disease. Med Physical Education, Sofia 1989.

10. Bankov S. Manual muscular testing with the basics of kinesiology and pathogenesisology. Sofia 1987.

11. Luoma K, Riihimaki H, Luukkonen R, Raininko R, Viikari-Juntura E, Lamminen A. Low back pain in relation to lumbar disc degeneration. Spine 2000; 25: 487-492.

12. Karaneshev G. Theory and methodology of the physical education. Sofia 1991.

13. Manusov EG. Evaluation and diagnosis of low back pain. Prim Care 2012; 39: 471-479.

14. Borenstein DG, Wiesel SW, Boden SD. Low back pain medical diagnosis and comprehensive management (2nd Edition). W.B. Saunders, Philadelphia 1995; 181-589.

15. Van Tulder MW, Scolten RJPM, Koes BW, Deyo RA. Non-steroidal anti-inflammatory drugs for low back pain. Spine 2000; 25: 2501-2513.

16. Choi BK, Verbeek JH, Tam WW, Jiang JY. Exercises for prevention of recurrences of low-back pain. Cochrane Database Systematic Rev 2010.

17. Carey TS, Garrett JM, Jackman A, Hadler N. Recurrence and care seeking after acute back pain: results of a longterm follow-up study. Med Care 1999; 37: 157-164.

18. Furlan AD, Imamura M, Dryden T, Irvin E. Massage for low-back pain. Cochrane Database Systematic Rev 2008.

19. Riazkova M. Practical clinical physiotherapy. Knowledge 1999; 14-16.

20. Zhelev V. Physiotherapy. Third part: specialized methods and methods in physiotherapy. Sofia 2011.

21. Todorov T, Todorov I, Mihailova M, Vladeva E. Contemporary physical therapy and disability disorder rehabilitation. Varna Medical Forum 2014.

\section{${ }^{*}$ Correspondence to}

Penka Petleshkova

Department of Obstetrics and Gynecology

Plovdiv Medical University

Bulgaria 